\title{
Monotone Iterative Method for Fractional Differential Equations with Integral Boundary Conditions
}

\author{
Shiying Song, ${ }^{1}$ Hongyu Li $\mathbb{D},{ }^{1}$ and Yumei Zou $\mathbb{D}^{2}$ \\ ${ }^{1}$ Department of Applied Mathematics, Shandong University of Science and Technology, Qingdao 266590, China \\ ${ }^{2}$ Department of Statistics and Finance, Shandong University of Science and Technology, Qingdao 266590, China
}

Correspondence should be addressed to Yumei Zou; sdzouym@126.com

Received 4 February 2020; Accepted 4 March 2020; Published 30 March 2020

Guest Editor: Chuanjun Chen

Copyright (c) 2020 Shiying Song et al. This is an open access article distributed under the Creative Commons Attribution License, which permits unrestricted use, distribution, and reproduction in any medium, provided the original work is properly cited.

In this paper, the existence of extremal solutions for fractional differential equations with integral boundary conditions is obtained by using the monotone iteration technique and the method of upper and lower solutions. The main equations studied are as follows: $\left\{\begin{array}{l}-D_{0+}^{\alpha} u(t)=f(t, u(t)), t \in[0,1], \\ u(0)=0, u(1)=\int_{0}^{1} u(t) \mathrm{d} A(t),\end{array}\right.$ where $D_{0+}^{\alpha}$ is the standard Riemann-Liouville fractional derivative of order $\alpha \in(1,2)$ and $A(t)$ is a positive measure function. Moreover, an example is given to illustrate the main results.

\section{Introduction}

The purpose of this paper is to study the following differential equations with integral boundary conditions:

$$
\left\{\begin{array}{l}
-D_{0+}^{\alpha} u(t)=f(t, u(t)), \quad t \in[0,1] \\
u(0)=0 \\
u(1)=\int_{0}^{1} u(t) \mathrm{d} A(t)
\end{array}\right.
$$

where $D_{0+}^{\alpha}$ is the standard Riemann-Liouville fractional derivative and $1<\alpha<2$ and $A(t)$ is a positive measure function, and it satisfies $\int_{0}^{1} G(t) \mathrm{d} A(t)<G(1)$.

Fractional differential equations have been widely used in physics, chemistry, aerodynamics, electrodynamics of complex media, and rheology of polymers [1-7]. As a result, various nonlinear functional analysis methods have been used to study the existence of solutions for differential equations [8-38]. However, it is difficult to obtain the exact solutions of fractional differential equations, so the monotone iteration method and the upper and lower solutions are generally used to obtain the approximate solutions of fractional differential problems. This method is also applicable to both initial value problems and boundary value problems for integer-order differential equations and differential systems [39-43]. In recent years, many scholars have used this method to study various fractional differential equation problems [44-55]. For example, in [47, 48, 52], the authors paid attention to the Riemann-Liouville fractional differential equations of order $\alpha \in(1,2)$, and in [44, $45,49,55]$, the authors considered the Riemann-Liouville fractional differential equation of order $\alpha \in(0,1)$, while in [46], the authors considered the boundary value problem with Riemann-Liouville fractional order $\alpha \in(2,3)$. Of course, some papers also use the monotone iterative method to deal with nonlinear Caputo fractional differential equations [56].

Based on the upper and lower solutions, this paper presents a method to prove the existence of solutions of Riemann-Liouville fractional differential equation (1). By using the monotone iteration technique coupled with the upper and lower solution method, a new comparison principle is established and the existence of the extremal solution of integral boundary value problems (1) is proved.

This paper is mainly divided into the following two parts: Section 2 mainly introduces the preparation of this article, and then in Section 3, the monotone sequence of solutions is constructed, and the main result of integral boundary value problems (1) is given. 


\section{Preliminaries}

In this section, we will briefly introduce some of the necessary definitions and results that will be used in the main results.

Definition 1 (see $[2,5])$. The fractional integral of order $\alpha>0$ of a function $u:(0, \infty) \longrightarrow R$ is given by

$$
I_{0+}^{\alpha} u(t)=\frac{1}{\Gamma(\alpha)} \int_{0}^{t}(t-s)^{\alpha-1} u(s) \mathrm{d} s,
$$

provided that the right-hand side is point-wise defined on $(0,+\infty)$.

Definition 2 (see $[2,5]$ ). The Riemann-Liouville fractional derivative of order $\alpha>0$ of a function $u:(0,+\infty) \longrightarrow \mathbb{R}$ is given by

$$
D_{0+}^{\alpha} u(t)=\frac{1}{\Gamma(n-\alpha)}\left(\frac{\mathrm{d}}{\mathrm{d} t}\right)^{n} \int_{0}^{t}(t-s)^{n-\alpha-1} u(s) \mathrm{d} s,
$$

where $n=[\alpha]+1,[\alpha]$ denotes the integer part of number $\alpha$, provided that the right-hand side is point-wise defined on $(0,+\infty)$.

Let $E=\left\{u \in C[0,1]: D_{0+}^{\alpha} u \in C[0,1]\right\}$ be endowed with the norm $\|u\|=\max \left\{\|u\|_{\infty},\left\|D_{0+}^{\alpha} u\right\|_{\infty}\right\} \quad$ in which $\|u\|_{\infty}=\max _{t \in[0,1]}|u(t)|$, and then $(E,\|\cdot\|)$ is a Banach space.

Definition 3. We say that $u \in E$ is an upper solution of (1) if it satisfies

$$
\left\{\begin{array}{l}
-D_{0+}^{\alpha} u(t) \geq f(t, u(t)), \quad t \in[0,1] \\
u(0)=0, \quad u(1) \geq \int_{0}^{1} u(t) \mathrm{d} A(t)
\end{array}\right.
$$

Definition 4. We say that $v \in E$ is a lower solution of (1) if it satisfies

$$
\left\{\begin{array}{l}
-D_{0+}^{\alpha} v(t) \leq f(t, v(t)), \quad t \in[0,1], \\
v(0)=0, \quad v(1) \leq \int_{0}^{1} v(t) \mathrm{d} A(t) .
\end{array}\right.
$$

Denote

$$
g(t)=\frac{\alpha-2}{\Gamma(\alpha-1)}+\sum_{k=1}^{+\infty} \frac{t^{k}}{\Gamma((k+1) \alpha-2)} .
$$

It is easy to check that (see $[57,58]$ )

$$
\begin{aligned}
g(0) & =\frac{\alpha-2}{\Gamma(\alpha-1)}<0, \\
g^{\prime}(t) & >0, \quad t \in[0,+\infty), \\
\lim _{t \longrightarrow+\infty} g(t) & =+\infty .
\end{aligned}
$$

Therefore, there exists a unique number $b^{*}>0$ such that

$$
g\left(b^{*}\right)=0 \text {. }
$$

Set $\quad G(t)=t^{\alpha-1} E_{\alpha, \alpha}\left(b t^{\alpha}\right), \quad$ where $\quad E_{\alpha, \alpha}(x)=$ $\sum_{k=0}^{+\infty}\left(x^{k} /(\Gamma((k+1) \alpha))\right)$ is the Mittag-Leffler function (see $[2,5])$.

In this article, we list the following assumption for convenience:

(H1): the parameter $b$ satisfies $b \in\left(0, b^{*}\right]$.

$(H 2): A(t)$ is a positive measure function and $0<G(1)-\int_{0}^{1} G(t) \mathrm{d} A(t)$.

(H3): assume that $w_{0}, v_{0} \in E$ are the upper and lower solutions of problem (1), respectively, and $v_{0}(t) \leq w_{0}(t), t \in[0,1]$.

$(H 4): f \in C([0,1] \times \mathbb{R}, \mathbb{R})$ and $f(t, w)-f(t, v) \geq$ $-b(w-v)$ for $v_{0}(t) \leq v \leq w \leq w_{0}(t), t \in[0,1]$.

Next, we will consider the following auxiliary linear boundary value problem:

$$
\left\{\begin{array}{l}
-D_{0+}^{\alpha} u(t)+b u(t)=y(t), \quad t \in[0,1], \\
u(0)=0, \\
u(1)=\int_{0}^{1} u(t) \mathrm{d} A(t)+c, \quad c \in \mathbb{R} .
\end{array}\right.
$$

Lemma 1. Suppose that (H1) and (H2) hold and $y \in C[0,1]$. Then, fractional boundary value problem (9) has the following unique solution:

$$
u(t)=\int_{0}^{1} K(t, s) y(s) \mathrm{d} s+\frac{c G(t)}{G(1)-\int_{0}^{1} G(t) \mathrm{d} A(t)},
$$

where

$$
\begin{aligned}
K(t, s) & =K_{0}(t, s)+G(t) h(s), \\
h(s) & =\frac{\int_{0}^{1} K_{0}(t, s) \mathrm{d} A(t)}{G(1)-\int_{0}^{1} G(t) \mathrm{d} A(t)}, \\
K_{0}(t, s) & =\frac{1}{G(1)} \begin{cases}G(t) G(1-s), & 0 \leq t \leq s \leq 1, \\
G(t) G(1-s)-G(t-s) G(1), & 0 \leq s \leq t \leq 1 .\end{cases}
\end{aligned}
$$

Proof. The main idea of Lemma 1 comes from [57]. By [2], we first find the solution of the fractional differential equations

$$
-D_{0+}^{\alpha} u(t)+b u(t)=y(t), \quad t \in[0,1]
$$

with two-point boundary condition

$$
\begin{aligned}
& u(0)=0, \\
& u(1)=a \in \mathbb{R},
\end{aligned}
$$

which can be expressed by

$$
u(t)=-\int_{0}^{t} G(t-s) y(s) \mathrm{d} s+c_{1} G(t)+c_{2} G^{\prime}(t) .
$$


Since $u(0)=0$, we have $c_{2}=0$. Then, by the condition $u(1)=a$, we calculated that

$$
c_{1}=\frac{\int_{0}^{1} G(1-s) y(s) \mathrm{d} s+a}{G(1)} .
$$

Therefore, the solution of (12) and (13) is

$$
\begin{aligned}
u(t) & =-\int_{0}^{t} G(t-s) y(s) \mathrm{d} s+\frac{\int_{0}^{1} G(1-s) y(s) \mathrm{d} s+a}{G(1)} G(t) \\
& =\int_{0}^{1} K_{0}(t, s) y(s) \mathrm{d} s+\frac{u(1)}{G(1)} G(t) .
\end{aligned}
$$

Next, we consider problem (9). Integrating equality (16) with respect to $A(t)$, we have

$$
\begin{aligned}
\int_{0}^{1} u(t) \mathrm{d} A(t)= & \int_{0}^{1} \int_{0}^{1} K_{0}(t, s) y(s) \mathrm{d} s \mathrm{~d} A(t) \\
& +\frac{u(1) \int_{0}^{1} G(t) \mathrm{d} A(t)}{G(1)} .
\end{aligned}
$$

Making use of the condition $u(1)=\int_{0}^{1} u(t) \mathrm{d} A(t)+c$ in the above equality yields

$$
u(1)-c=\int_{0}^{1} \int_{0}^{1} K_{0}(t, s) y(s) \mathrm{d} s \mathrm{~d} A(t)+\frac{u(1) \int_{0}^{1} G(t) \mathrm{d} A(t)}{G(1)},
$$

and then we get

$$
u(1)\left(1-\frac{\int_{0}^{1} G(t) \mathrm{d} A(t)}{G(1)}\right)=\int_{0}^{1} \int_{0}^{1} K_{0}(t, s) y(s) \mathrm{d} s \mathrm{~d} A(t)+c .
$$

Obviously,

$$
u(1)=\frac{G(1) \int_{0}^{1} \int_{0}^{1} K_{0}(t, s) y(s) \mathrm{d} s \mathrm{~d} A(t)+c G(1)}{G(1)-\int_{0}^{1} G(t) \mathrm{d} A(t)} .
$$

Therefore,

$$
\begin{aligned}
u(t) & =\int_{0}^{1} K_{0}(t, s) y(s) \mathrm{d} s+\frac{G(t)\left(G(1) \int_{0}^{1} \int_{0}^{1} K_{0}(t, s) y(s) \mathrm{d} s \mathrm{~d} A(t)+c G(1)\right)}{G(1)\left(G(1)-\int_{0}^{1} G(t) \mathrm{d} A(t)\right)} \\
& =\int_{0}^{1} K_{0}(t, s) y(s) \mathrm{d} s+\frac{G(t)\left(\int_{0}^{1} \int_{0}^{1} K_{0}(t, s) y(s) \mathrm{d} s \mathrm{~d} A(t)+c\right)}{G(1)-\int_{0}^{1} G(t) \mathrm{d} A(t)} \\
& =\int_{0}^{1} K_{0}(t, s) y(s) \mathrm{d} s+\frac{\int_{0}^{1} G(t)\left(\int_{0}^{1} K_{0}(t, s) \mathrm{d} A(t)\right) y(s) \mathrm{d} s}{G(1)-\int_{0}^{1} G(t) \mathrm{d} A(t)}+\frac{c G(t)}{G(1)-\int_{0}^{1} G(t) \mathrm{d} A(t)} \\
& =\int_{0}^{1} K_{0}(t, s) y(s) \mathrm{d} s+\frac{c G(t)}{G(1)-\int_{0}^{1} G(t) \mathrm{d} A(t)} .
\end{aligned}
$$

The proof is completed.

Lemma 2. Suppose that (H1) and (H2) hold, and $u \in E$ satisfies

$$
\left\{\begin{array}{l}
-D_{0+}^{\alpha} u(t)+b u(t) \geq 0, \\
u(0)=0, \quad u(1) \geq \int_{0}^{1} u(t) \mathrm{d} A(t) .
\end{array}\right.
$$

Then, for $t \in[0,1], u(t) \geq 0$.

Proof. Let $y(t)=-D_{0+}^{\alpha} u(t)+b u(t) \quad$ and $\quad c=u(1)-$ $\int_{0}^{1} u(t) \mathrm{d} A(t)$. From (12), we have $y(t) \geq 0, c \geq 0$, and

$$
\left\{\begin{array}{l}
-D_{0+}^{\alpha} u(t)+b u(t)=y(t), \\
u(0)=0, \quad u(1)=\int_{0}^{1} u(t) \mathrm{d} A(t)+c .
\end{array}\right.
$$

By Lemma 1, we obtain that problem (23) has unique solution $u$, which can be expressed as follows:

$$
u(t)=\int_{0}^{1} K(t, s) y(s) \mathrm{d} s+\frac{c G(t)}{G(1)-\int_{0}^{1} G(t) \mathrm{d} A(t)} .
$$

From [57], it follows that $K_{0}(t, s) \geq 0$ for $t, s \in[0,1]$. This together with $(H 1)$ and $(H 2)$ yields 
$K(t, s)=K_{0}(t, s)+G(t) \frac{\int_{0}^{1} K_{0}(t, s) \mathrm{d} A(t)}{G(1)-\int_{0}^{1} G(t) \mathrm{d} A(t)} \geq 0$,

$$
t, s \in[0,1] .
$$

Hence, we conclude that

$$
u(t) \geq \frac{c G(t)}{G(1)-\int_{0}^{1} G(t) \mathrm{d} A(t)} \geq 0, \quad t \in[0,1],
$$

which completes the proof.

\section{Main Results}

For $v_{0}, w_{0} \in E$ with $v_{0}(t) \leq w_{0}(t)$ for $t \in[0,1]$, we denote an ordered interval:

$$
\left[v_{0}, w_{0}\right]=\left\{u \in E: v_{0}(t) \leq u(t) \leq w_{0}(t), \quad t \in[0,1]\right\} .
$$

Theorem 1. Suppose (H1)-(H4) hold, and then there exist monotone iterative sequences $\left\{v_{n}\right\},\left\{w_{n}\right\} \subset\left[v_{0}, w_{0}\right]$ such that $v_{n} \longrightarrow v^{*}, w_{n} \longrightarrow w^{*}$ as $n \longrightarrow \infty$ uniformly in $\left[v_{0}, w_{0}\right]$, and $v^{*}, w^{*}$ are a minimal and a maximal solution of (1) in $\left[v_{0}, w_{0}\right]$, respectively.
Proof. For $v_{n-1}, w_{n-1} \in E, n \geq 1$, we define two sequences $\left\{v_{n}\right\},\left\{w_{n}\right\} \subset E$ satisfying the following fractional differential equation:

$$
\left\{\begin{array}{l}
-D_{0+}^{\alpha} v_{n}(t)+b v_{n}(t)=f\left(t, v_{n-1}(t)\right)+b v_{n-1}(t), \quad t \in[0,1], \\
v_{n}(0)=0, v_{n}(1)=\int_{0}^{1} v_{n}(t) \mathrm{d} A(t),
\end{array}\right.
$$

$$
\left\{\begin{array}{l}
-D_{0+}^{\alpha} w_{n}(t)+b w_{n}(t)=f\left(t, w_{n-1}(t)\right)+b w_{n-1}(t), \\
t \in[0,1], \\
w_{n}(0)=0, \quad w_{n}(1)=\int_{0}^{1} w_{n}(t) \mathrm{d} A(t) .
\end{array}\right.
$$

By consideration of Lemma 1, for any $n \geq 1$, problems (28) and (29) have a unique solution $v_{n+1}(t), w_{n+1}(t)$, respectively, which are well defined.

Firstly, we need to show that, for any $t \in[0,1]$, $v_{0}(t) \leq v_{1}(t) \leq w_{1}(t) \leq w_{0}(t)$. Let $x(t)=v_{1}(t)-v_{0}(t)$, and the definition of $v_{1}(t)$ together with $(H 3)$ yields

$$
\left\{\begin{array}{l}
-D_{0+}^{\alpha} x(t)+b x(t)=-D_{0+}^{\alpha}\left(v_{1}(t)-v_{0}(t)\right)+b\left(v_{1}(t)-v_{0}(t)\right)=-D_{0+}^{\alpha} v_{1}(t)+b v_{1}(t)-\left[-D_{0+}^{\alpha} v_{0}(t)+b v_{0}(t)\right] \\
\geq f\left(t, v_{0}(t)\right)+b v_{0}(t)-f\left(t, v_{0}(t)\right)-b v_{0}(t)=0, \\
x(0)=v_{1}(0)-v_{0}(0)=0 \\
x(1)=v_{1}(1)-v_{0}(1) \geq \int_{0}^{1} v_{1}(t) \mathrm{d} A(t)-\int_{0}^{1} v_{0}(t) \mathrm{d} A(t)=\int_{0}^{1} x(t) \mathrm{d} A(t) .
\end{array}\right.
$$

In the light of Lemma 2, we have $x(t) \geq 0, t \in[0,1]$, namely, $v_{1}(t) \geq v_{0}(t)$. Similarly, it can be shown that $w_{0}(t) \geq w_{1}(t), t \in[0,1]$.

Secondly, we make $h(t)=w_{1}(t)-v_{1}(t)$. From $(H 4)$, we get

$$
\begin{aligned}
-D_{0+}^{\alpha} h(t)= & f\left(t, w_{0}(t)\right)-b\left[w_{1}(t)-w_{0}(t)\right]-f\left(t, v_{0}(t)\right) \\
& +b\left[v_{1}(t)-v_{0}(t)\right] \\
\geq & -b\left[w_{0}(t)-v_{0}(t)\right]-b\left[w_{1}(t)-w_{0}(t)\right] \\
& +b\left[v_{1}(t)-v_{0}(t)\right] \\
= & -b h(t) .
\end{aligned}
$$

Also, $h(0)=0$ and $h(1)=\int_{0}^{1} h(t) \mathrm{d} A(t)$. Thus, Lemma 2 implies that, for any $t \in[0,1], w_{1}(t) \geq v_{1}(t)$.

Thirdly, we prove that $w_{1}, v_{1}$ are upper and lower solutions of problem (1), respectively. Note that

$$
\begin{aligned}
-D_{0+}^{\alpha} v_{1}(t)= & f\left(t, v_{0}(t)\right)+b v_{0}(t)-b v_{1}(t)-f\left(t, v_{1}(t)\right) \\
& +f\left(t, v_{1}(t)\right) \\
= & f\left(t, v_{0}(t)\right)-f\left(t, v_{1}(t)\right)+f\left(t, v_{1}(t)\right) \\
& -b\left[v_{1}(t)-v_{0}(t)\right] \\
\leq & b\left[v_{1}(t)-v_{0}(t)\right]+f\left(t, v_{1}(t)\right)-b\left[v_{1}(t)-v_{0}(t)\right] \\
= & f\left(t, v_{1}(t)\right) .
\end{aligned}
$$

And by assumption (H4), $\quad v_{1}(0)=0$ and $v_{1}(1)=\int_{0}^{1} v_{1}(t) \mathrm{d} A(t)$. This shows that $v_{1}$ is a lower solution of problem (1). Similarly, we can infer that $w_{1}$ is an upper solution of (1).

Using mathematical induction, it is easy to verify that

$$
\begin{aligned}
v_{0}(t) & \leq v_{1}(t) \leq \cdots \leq v_{n}(t) \leq \cdots \leq w_{n}(t) \leq \cdots \\
& \leq w_{1}(t) \leq w_{0}(t) .
\end{aligned}
$$


Clearly, it is easy to conclude that $\left\{v_{n}\right\}$ and $\left\{w_{n}\right\}$ are uniformly bounded in $C[0,1]$. Moreover, by Lemma 1 , problems (28) and (29) are equivalent to the following integral equation:

$$
\begin{aligned}
& v_{n}(t)=\int_{0}^{1} K(t, s)\left[f\left(s, v_{n-1}(s)+b v_{n-1}(s)\right] \mathrm{d} s,\right. \\
& w_{n}(t)=\int_{0}^{1} K(t, s)\left[f\left(s, w_{n-1}(s)+b w_{n-1}(s)\right] \mathrm{d} s,\right.
\end{aligned}
$$

respectively. Therefore, the continuity of the functions $K(t, s)$ allows us to conclude that $\left\{v_{n}\right\}$ and $\left\{w_{n}\right\}$ are equicontinuous in $C[0,1]$. Using (28) and (29) again, we know that $\left\{D_{0+}^{\alpha} v_{n}\right\}$ and $\left\{D_{0+}^{\alpha} w_{n}\right\}$ are uniformly bounded and equicontinuous in $C[0,1]$. So, $\left\{v_{n}\right\}$ and $\left\{w_{n}\right\}$ are uniformly bounded and equicontinuous in $E$. Using the standard arguments, we have $\left\{v_{n}\right\}$ and $\left\{w_{n}\right\}$ converging, say, to $v^{*}$ and $w^{*}$, uniformly on $[0,1]$, respectively. That is,

$$
\begin{aligned}
\lim _{n \longrightarrow \infty} v_{n}(t) & =v^{*}(t), \\
\lim _{n \longrightarrow \infty} w_{n}(t) & =w^{*}(t), \\
t & \in[0,1] .
\end{aligned}
$$

Furthermore, $v^{*}(t)$ and $w^{*}(t)$ are the solutions of problem (1), and $v_{0} \leq v^{*} \leq w^{*} \leq w_{0}$ on $[0,1]$.

Next, we need to prove that $v^{*}$ and $w^{*}$ are extremal solutions of $(1)$ in $\left[v_{0}, w_{0}\right]$. Let $u \in\left[v_{0}, w_{0}\right]$ be any solution of problem (1). We assume that $v_{m}(t) \leq u(t) \leq w_{m}(t), t \in[0,1]$ for some $m$. Take $p(t)=u(t)-v_{m+1}(t)$, $q(t)=w_{m+1}(t)-u(t)$. Then, by assumption $(H 4)$, we obtain

$$
\begin{aligned}
& \left\{\begin{array}{l}
-D_{0+}^{\alpha} p(t) \geq-b p(t), \quad t \in[0,1], \\
p(0)=0, \quad p(1)=\int_{0}^{1} p(t) \mathrm{d} A(t),
\end{array}\right. \\
& \left\{\begin{array}{l}
-D_{0+}^{\alpha} q(t) \geq-b q(t), \quad t \in[0,1], \\
q(0)=0, \quad q(1)=\int_{0}^{1} q(t) \mathrm{d} A(t) .
\end{array}\right.
\end{aligned}
$$

By Lemma 2, we have

$$
v_{m+1}(t) \leq u(t) \leq w_{m+1}(t), \quad t \in[0,1] .
$$

Applying mathematical induction, one has $v_{n}(t) \leq u(t) \leq w_{n}(t)$ on $[0,1]$ for any $n$. Taking the limit, we conclude $v^{*}(t) \leq u(t) \leq w^{*}(t), t \in[0,1]$. The proof is complete.

Example 1. Consider the following problem:

$$
\left\{\begin{array}{l}
-D_{0+}^{4 / 3} u(t)=-\frac{u^{3}(t)}{10}+\frac{\sin t}{10}, \quad t \in[0,1] \\
u(0)=0 \\
u(1)=\int_{0}^{1} u(t) \mathrm{d} t
\end{array}\right.
$$

where $\alpha=4 / 3, A(t)=t$, and $f(t, u)=-\left(u^{3} / 10\right)+(\sin t / 10)$.
Taking $v_{0}(t)=-t^{1 / 3}$ and $w_{0}(t)=t^{1 / 3}$, we have

$$
\begin{aligned}
& \left\{\begin{array}{l}
-D_{0+}^{4 / 3} v_{0}(t)=0 \leq \frac{t}{10}+\frac{\sin t}{10}=f\left(t, v_{0}(t)\right), \quad t \in[0,1], \\
v_{0}(0)=0, v_{0}(1)=-1<-\frac{3}{4}=\int_{0}^{1} v_{0}(t) \mathrm{d} t,
\end{array}\right. \\
& \left\{\begin{array}{l}
-D_{0+}^{4 / 3} w_{0}(t)=0 \geq-\frac{t}{10}+\frac{\sin t}{10}=f\left(t, w_{0}(t)\right), \quad t \in[0,1], \\
w_{0}(0)=0, w_{0}(1)=1>\frac{3}{4}=\int_{0}^{1} w_{0}(t) \mathrm{d} t,
\end{array}\right.
\end{aligned}
$$

which shows that $v_{0}(t)$ and $w_{0}(t)$ are a lower and an upper solution of (38), respectively, and $v_{0}(t) \leq w_{0}(t)$. So, (H3) holds.

Using the strictly monotone increasing property of gamma function $\Gamma(\cdot)$ on $[2,+\infty)$, we have

$$
\begin{aligned}
g(t)= & -\frac{2}{3 \Gamma(1 / 3)}+\sum_{k=1}^{\infty} \frac{t^{k}}{\Gamma((4 k / 3)-(2 / 3))}=-\frac{2}{3 \Gamma(1 / 3)} \\
& +\frac{t}{\Gamma(2 / 3)}+\sum_{k=2}^{\infty} \frac{t^{k}}{\Gamma(((4(k-2)) / 3)+2)} \\
& \leq-\frac{2}{3 \Gamma(1 / 3)}+\frac{t}{\Gamma(2 / 3)}+\sum_{k=2}^{\infty} \frac{t^{k}}{\Gamma(k)}=-\frac{2}{3 \Gamma(1 / 3)} \\
& +\frac{t}{\Gamma(2 / 3)}+t\left(e^{t}-1\right), \quad t \in[0,+\infty) .
\end{aligned}
$$

By MATLAB, we obtain $g(3 / 10)<-0.0568$. Therefore, $b^{*}>b:=(3 / 10)$. In addition, we have

$$
f(t, w)-f(t, v)=-\left[\frac{w^{3}}{10}-\frac{v^{3}}{10}\right] \geq-\frac{3}{10}(w-v),
$$

for $v_{0}(t) \leq v \leq w \leq w_{0}(t), t \in[0,1]$. Hence, $(H 1)$ and $(H 4)$ hold.

Note that $G(t)=\sum_{k=0}^{+\infty}(3 / 10)^{k}\left(t^{(4 k+1) / 3} /(\Gamma((4 k+4) / 3))\right)$. Then,

$$
\begin{aligned}
\int_{0}^{1} G(t) \mathrm{d} t & =\sum_{k=0}^{+\infty}\left(\frac{3}{10}\right)^{k} \frac{\int_{0}^{1} t^{(4 k+1) / 3)} \mathrm{d} t}{\Gamma((4 k+4) / 3)} \\
& =\sum_{k=0}^{+\infty}\left(\frac{3}{10}\right)^{k} \frac{1}{((4 k+4) / 3) \Gamma(((4 k+4) / 3))} \\
& <\sum_{k=0}^{+\infty}\left(\frac{3}{10}\right)^{k} \frac{1}{\Gamma((4 k+4) / 3)}=G(1) .
\end{aligned}
$$

It shows that (H2) holds. Thus, Theorem 1 ensures that problem (38) has extremal solutions in $\left[v_{0}, w_{0}\right]$. 


\section{Data Availability}

The data used to support the findings of this study are included within the article.

\section{Conflicts of Interest}

The authors declare that they have no conflicts of interest.

\section{Acknowledgments}

This project was supported by the National Natural Science Foundation of China (51774197 and 11801322), the Shandong Natural Science Foundation (ZR2018MA011), and SDUST graduate innovation project (SDKDYC190238).

\section{References}

[1] P. Agarwal, S. Jain, and T. Mansour, "Further extended caputo fractional derivative operator and its applications," Russian Journal of Mathematical Physics, vol. 24, no. 4, pp. 415-425, 2017.

[2] A. A. Kilbas, H. M. Srivastava, and J. J. Trujillo, Theory and Applications of Fractional Differential Equations, Elsevier, Amsterdam, the Netherlands, 2006.

[3] C. Fu, C. Lu, and H. Yang, "Time-space fractional $(2+1)$ dimensional nonlinear Schrodinger equation for envelope gravity waves in baroclinic atmosphere and conservation laws as well as exact solutions," Advances in Difference Equations, vol. 2018, no. 1, Article ID 56, 2018.

[4] M. Guo, H. Dong, J. Liu, and H. Yang, "The time-fractional mZK equation for gravity solitary waves and solutions using sech-tanh and radial basic function method," Nonlinear Analysis: Modelling and Control, vol. 24, no. 1, pp. 1-19, 2019.

[5] Podlubny, Fractional Differential Equations, Mathematics in Science and Engineering, Academic Press, Cambridge, MA, USA, 1999.

[6] C. Chen, K. Li, Y. Chen, and Y. Huang, "Two-grid finite element methods combined with Crank-Nicolson scheme for nonlinear Sobolev equations," Advances in Computational Mathematics, vol. 45, no. 2, pp. 611-630, 2019.

[7] C. Chen, H. Liu, X. Zheng et al., "A two-grid MMOC finite element method for nonlinear variable-order time-fractional mobile/immobile advection-diffusion equations," Computers and Mathematics with Applications, 2019, In Press.

[8] W. Cheng, J. Xu, Y. Cui, and Q. Ge, "Positive solutions for a class of fractional difference systems with coupled boundary conditions," Advances in Difference Equations, vol. 2019, Article ID 249, 2019.

[9] W. Cheng, J. Xu, D. O’Regan, and Y. Cui, "Positive solutions for a nonlinear discrete fractional boundary value problem with a $p$-Laplacian operator," Journal of Applied Analysis and Computation, vol. 9, no. 5, pp. 1959-1972, 2019.

[10] J. He, X. Zhang, L. Liu, Y. Wu, and Y. Cui, “A singular fractional Kelvin-Voigt model involving a nonlinear operator and their convergence properties," Boundary Value Problems, vol. 2019, Article ID 112, 2019.

[11] J. He, X. Zhang, L. Liu, and Y. Wu, "Existence and nonexistence of radial solutions of the Dirichlet problem for a class of general $k$-Hessian equations," Nonlinear Analysis: Modelling and Control, vol. 23, no. 4, pp. 475-492, 2018.
[12] H. Li and J. Zhang, "Positive solutions for a system of fractional differential equations with two parameters," Journal of Function Spaces, vol. 2018, Article ID 1462505, 9 pages, 2018.

[13] D. Li, F. Chen, and Y. An, "Existence and multiplicity of nontrivial solutions for nonlinear fractional differential systems with $p$-Laplacian via critical point theory," Mathematical Methods in the Applied Sciences, vol. 41, no. 8, pp. 3197-3212, 2018.

[14] Z. Lou and J. Xu, "Symmetric results of a Hénon type elliptic equation," Applied Mathematics Letters, vol. 96, pp. 54-60, 2019.

[15] B. Meng, "Minimal thinness with respect to the Schrodinger operator and its applications on singular Schrodinger-type boundary value problems," Boundary Value Problems, vol. 2019, Article ID 91, 2019.

[16] S. Meng and Y. Cui, "Multiplicity results to a conformable fractional differential equations involving integral boundary condition," Complexity, vol. 2019, Article ID 8402347, 8 pages, 2019.

[17] T. Ren, H. Xiao, Z. Zhou, and X. Zhang, "The iterative scheme and the convergence analysis of unique solution for a singular fractional differential equation from the eco-economic complex systems co-evolution process," Complexity, vol. 2019, Article ID 9278056, 15 pages, 2019.

[18] U. Riaz, A. Zada, Z. Ali, Y. Cui, and J. Xu, "Analysis of coupled systems of implicit impulsive fractional differential equations involving Hadamard derivatives," Advances in Difference Equations, vol. 2019, Article ID 226, 2019.

[19] Q. Song and Z. Bai, "Positive solutions of fractional differential equations involving the Riemann-Stieltjes integral boundary condition," Advances in Difference Equations, vol. 2018, Article ID 183, 2018.

[20] S. Song and Y. Cui, "Existence of solutions for integral boundary value problems of mixed fractional differential equations under resonance," Boundary Value Problems, vol. 2020, Article ID 23, 2020.

[21] F. Wang and Y. Cui, "Positive solutions for an infinite system of fractional order boundary value problems," Advances in Difference Equations, vol. 2019, Article ID 169, 2019.

[22] Y. Wang, "Positive solutions for a class of two-term fractional differential equations with multipoint boundary value conditions," Advances in Difference Equations, vol. 2019, Article ID 304, 2019.

[23] Y. Wang, "Necessary conditions for the existence of positive solutions to fractional boundary value problems at resonance," Applied Mathematics Letters, vol. 97, pp. 34-40, 2019.

[24] Y. Wang, "Existence of uniqueness and nonexistence results of positive solution for fractional differential equations integral boundary value problems," Journal of Function Spaces, vol. 2018, Article ID 1547293, 7 pages, 2018.

[25] M. Xu and Z. Han, "Positive solutions for integral boundary value problem of two-term fractional differential equations," Boundary Value Problems, vol. 2018, Article ID 100, 2018.

[26] C. Zhai, W. Wang, and H. Li, "A uniqueness method to a new Hadamard fractional differential system with four-point boundary conditions," Journal of Inequalities and Applications, vol. 2018, Article ID 207, 2018.

[27] H. Zhang, Y. Li, and J. Xu, "Positive solutions for a system of fractional integral boundary value problems involving Hadamard-type fractional derivatives," Complexity, vol. 2019, Article ID 2671539, 11 pages, 2019.

[28] J. Zhang, G. Zhang, and H. Li, "Positive solutions of secondorder problem with dependence on derivative in nonlinearity under Stieltjes integral boundary condition," Electronic 
Journal of Qualitative Theory of Differential Equations, vol. 2018, Article ID 4, 2018.

[29] L. Zhang, Y. Zhao, and S. Sun, "Lyapunov inequalities of left focal $q$-difference boundary value problems and applications," Advances in Difference Equations, vol. 2019, Article ID 131, 2018.

[30] X. Zhang, J. Xu, J. Jiang, Y. Wu, and Y. Cui, “The convergence analysis and uniqueness of blow-up solutions for a Dirichlet problem of the general $k$-Hessian equations," Applied Mathematics Letters, vol. 102, Article ID 106124, 2020.

[31] X. Zhang, L. Liu, Y. Wu, and Y. Cui, "A sufficient and necessary condition of existence of blow-up radial solutions for a $k$-Hessian equation with a nonlinear operator," Nonlinear Analysis: Modelling and Control, vol. 25, pp. 126-143, 2020.

[32] X. Zhang, J. Jiang, Y. Wu, and Y. Cui, "The existence and nonexistence of entire large solutions for a quasilinear Schrodinger elliptic system by dual approach," Applied Mathematics Letters, vol. 100, Article ID 106018, 2020.

[33] X. Zhang, J. Jiang, Y. Wu, and Y. Cui, "Existence and asymptotic properties of solutions for a nonlinear Schrödinger elliptic equation from geophysical fluid flows," Applied Mathematics Letters, vol. 90, pp. 229-237, 2019.

[34] X. Zhang, L. Liu, Y. Wu, and Y. Cui, "The existence and nonexistence of entire large solutions for a quasilinear Schrödinger elliptic system by dual approach," Journal of Mathematical Analysis and Applications, vol. 464, no. 2, pp. 1089-1106, 2018.

[35] X. Zhang, L. Liu, Y. Wu, and Y. Cui, "Existence of infinitely solutions for a modified nonlinear Schrodinger equation via dual approach," Electronic Journal of Differential Equations, vol. 2018, Article ID 147, 15 pages, 2018.

[36] X. Zhang, Y. Wu, and Y. Cui, "Existence and nonexistence of blow-up solutions for a Schrödinger equation involving a nonlinear operator," Applied Mathematics Letters, vol. 82, pp. 85-91, 2018.

[37] X. Zhang, L. Liu, and Y. Wu, "Multiple positive solutions of a singular fractional differential equation with negatively perturbed term," Mathematical and Computer Modelling, vol. 55, no. 3-4, pp. 1263-1274, 2012.

[38] Y. Zou, "Positive solutions for a fractional boundary value problem with a perturbation term," Journal of Function Spaces, vol. 2018, Article ID 9070247, 5 pages, 2018.

[39] G. S. Ladde, V. Lakshmikantham, and A. S. Vatsala, Monotone Iterative Techniques for Nonlinear Differential Equations, Pitman, Boston, MA, USA, 1985.

[40] Y. Cui and Y. Zou, "Existence of solutions for second-order integral boundary value problems," Nonlinear Analysis: Modelling and Control, vol. 21, no. 6, pp. 828-838, 2016.

[41] Y. Cui and Y. Zou, "Monotone iterative technique for $(k, n-k)$ conjugate boundary value problems," Electronic Journal of Qualitative Theory of Differential Equations, vol. 2015, Article ID 69, 2015.

[42] G. Wang, "Monotone iterative technique for boundary value problems of a nonlinear fractional differential equation with deviating arguments," Journal of Computational and Applied Mathematics, vol. 236, no. 9, pp. 2425-2430, 2012.

[43] G. Wang, Z. Bai, and L. Zhang, "Successive iterations for unique positive solution of a nonlinear fractional $q$-integral boundary value problem," Journal of Applied Analysis and Computation, vol. 9, pp. 1204-1215, 2019.

[44] Z. Bai, S. Zhang, S. Sun, and C. Yin, "Monotone iterative method for fractional differential equations," Electronic
Journal of Differential Equations, vol. 2016, Article ID 6, 8 pages, 2016.

[45] P. Chen and Y. Kong, "Monotone iterative technique for periodic boundary value problem of fractional differential equation in Banach spaces," International Journal of Nonlinear Sciences and Numerical Simulation, vol. 20, no. 5, pp. 595-599, 2019.

[46] Y. Cui, Q. Sun, and X. Su, "Monotone iterative technique for nonlinear boundary value problems of fractional order $p \in(2,3]$." Advances in Difference Equations, vol. 2017, Article ID 248, 2017.

[47] Y. Cui and Y. Zou, "Existence results and the monotone iterative technique for nonlinear fractional differential systems with coupled four-point boundary value problems," Abstract and Applied Analysis, vol. 2014, Article ID 242591, 6 pages, 2014.

[48] Y. He, "Existence results and the monotone iterative technique for nonlinear fractional differential systems involving fractional integral boundary conditions," Advances in Difference Equations, vol. 2017, Article ID 264, 2017.

[49] Y. Ding and Y. Li, "Monotone iterative technique for periodic problem involving Riemann-Liouville fractional derivatives in Banach spaces," Boundary Value Problems, vol. 2018, Article ID 119, 2018

[50] S. Meng and Y. Cui, "The extremal solution to conformable fractional differential equations involving integral boundary condition," Mathematics, vol. 7, p. 186, 2019.

[51] T. Ren, S. Li, X. Zhang, and L. Liu, "Maximum and minimum solutions for a nonlocal $p$-Laplacian fractional differential system from eco-economical processes," Boundary Value Problems, vol. 2017, Article ID 118, 2017.

[52] B. Tang, J. Zhao, and Z. Liu, "Monotone iterative method for two-point fractional boundary value problems," Advances in Difference Equations, vol. 2018, Article ID 182, 2018.

[53] J. Wu, X. Zhang, L. Liu, Y. Wu, and Y. Cui, "The convergence analysis and error estimation for unique solution of a $p$-Laplacian fractional differential equation with singular decreasing nonlinearity," Boundary Value Problems, vol. 2018, Article ID 82, 2018.

[54] J. Wu, X. Zhang, L. Liu, Y. Wu, and Y. Cui, "Convergence analysis of iterative scheme and error estimation of positive solution for a fractional differential equation," Mathematical Modelling and Analysis, vol. 23, no. 4, pp. 611-626, 2018.

[55] W. Zhang, Z. Bai, and S. Sun, "Extremal solutions for some periodic fractional differential equations," Advances in Difference Equations, vol. 2016, Article ID 179, 2016.

[56] M. Al-Refai and M. Ali Hajji, "Monotone iterative sequences for nonlinear boundary value problems of fractional order," Nonlinear Analysis: Theory, Methods \& Applications, vol. 74, no. 11, pp. 3531-3539, 2011.

[57] Y. Wang and L. Liu, "Positive properties of the green function for two-term fractional differential equations and its application," The Journal of Nonlinear Sciences and Applications, vol. 10, no. 4, pp. 2094-2102, 2017.

[58] Y. Wang and L. Liu, "Positive solutions for a class of fractional 3-point boundary value problems at resonance," Advances in Difference Equations, vol. 2017, Article ID 7, 2017. 